\title{
FORMACIÓN EN COMPETENCIAS ESPECÍFICAS PARA LA INDUSTRIA DEL SOFTWARE COLOMBIANO. EXPERIENCIAS DEL USO DEL APRENDIZAJE BASADO EN PROYECTOS
}

\section{SPECIFIC SKILLS TRAINING FOR COLOMBIAN SOFTWARE INDUSTRY. EXPERIENCE THE USE OF PROJECT-B ASED LEARNING}

\author{
PhD(c) Jorge Eliecer Giraldo Plaza, MSc. Maryem Aliria Ruiz Nuñez. \\ MSc. Claudia Alejadra Rosero Noguera, Esp. Luis Norberto Zapata Puerta. \\ Politécnico Colombiano Jaime Isaza Cadavid. \\ Facultad de Ingenierías. Sede Poblado, Medellín, Antioquia, Colombia. \\ E-mail: \{jegiraldo, mruiz, carosero, lnzapata\}@elpoli.edu.co
}

\begin{abstract}
Resumen: Se presenta una aproximación metodológica de la formación por competencias para sector de la industria del software mediante la aplicación de un enfoque de aprendizaje basado en proyectos pedagógicos integradores, con el fin de documentar la experiencia de formación de estudiantes en el área de los sistemas de información y presentar los objetivos alcanzados en términos de proyectos realizados y empresas impactadas.
\end{abstract}

Palabras clave: Competencias, proyectos integradores, aprendizaje, industria, software.

\begin{abstract}
A methodological based skills approach for training students in the sector of the software industry by implementing a system based on educational projects integrators in order to document the experience of training students in the area of information systems approach It presents the objectives achieved in terms of projects and businesses impacted
\end{abstract}

Keywords: Skills, integrators projects, learning, industry, software.

\section{INTRODUCCIÓN}

El aprendizaje basado en competencias busca que el estudiante genera las capacidades para la correcta utilización de los saberes con el fin de diseñar soluciones a necesidades de su área de desempeño (Turcio y Palacios, 2015).

El estilo de aprendizaje desempeña un papel importante en la formación del individuo, ya que se deben garantizar que realice actividades con destreza, según lo determina la industria (Spitzberg, 1983). En este caso el aprendizaje basado en proyectos, es el estilo apropiado, pues considera las experiencias prácticas útiles para enlazar conocimientos con productos y a su vez con ambientes reales de trabajo (Gonçalves, 2014).

La Facultad de Ingenierías del Politécnico Colombiano Jaime Isaza Cadavid, ofrece actualmente 2 programas de técnicas profesionales mediante aprendizaje basado en competencias, en el área de Sistemas de información, estas son: Técnica Profesional en programación de sistemas de información y Técnica profesional en soporte de sistemas de información.

Los programas académicos mencionados en su denominación académica abarcan áreas de desempeño provenientes del cuerpo de 
conocimientos de disciplinas tales como: sistemas de información, ingeniería de software y aplicación de las tecnologías de la información a las necesidades de las organizaciones.

Sistemas de información es una disciplina que relaciona los sistemas y la organización a la cual ellos sirven. Forma parte de las cinco disciplinas relacionadas con el área de computación propuesta por la ACM (2005).

Este documento presenta la aplicación del aprendizaje basado en competencias en la industria del software y sistemas de información. El caso de estudio se basa en la población de estudiantes del primer semestre de la Técnica Profesional en Soporte de Sistemas de Información.

Se propone un enfoque basado en proyectos, que consiste el diseño de un proyecto pedagógico integrador para cada uno de los semestres, donde se busca impactar una población y un sector de aplicación.

Por lo anterior, las experiencias van enfocadas a reducir la brecha de formación de los estudiantes y fortalecer la relación con las empresas de la industria afectada, priorizando la participación del estudiante en casos reales y su participación en equipos de trabajo colaborativo y auto-dirigidos.

El documento se estructura de la siguiente manera. En la sección 2, se presenta el marco conceptual relacionado, en la sección 3 los trabajos relacionados con la formación en competencias específicas y aprendizaje basado en proyectos, se presenta la metodología propuesta, En la sección 4 se detalla la metodología de formación propuesta. Para dar luego dar paso en la sección 5, a la validación y análisis de resultados; por último en la sección 6 , se presentan las conclusiones generales de documento y trabajos futuros.

\section{MARCO CONCEPTUAL}

\subsection{Formación basada en competencias}

El Aprendizaje Basado en Competencias (ABC) para la formación profesional es en la actualidad una prospectiva y una práctica para formar con pertinencia al educando con base en criterios de competencias profesionales, referenciadas con proyectos de formación por competencias nacionales e internacionales, incluidas las del siglo XXI (Severin, 2011).
La formación basada en competencias comprende las siguientes caracterís ticas:

- Las competencias pueden ser en algunos casos útiles $o$ inútiles, de acuerdo al contexto de ejecución.

- La ejecución de la competencia determina su nivel de pertinencia y efectividad, de esta manera su implementación se relaciona directamente con un ambiente real de ejecución.

- La evaluación de las competencias tienen niveles graduales asimilación por parte del individuo, por tanto puede llegarse a ser mas o menos componentes.

- Las competencias tienen como eje central el saber hacer las funciones específicas con calidad, más que el solo saber. Es decir busca que el dicente aprenda a ejecutar actividades correctas utilizando saberes específicos y comportamientos pertinentes.

En resumen el ABC significa diseñar los contenidos y las experiencias de aprendizaje después de establecer las competencias necesarias para un campo de acción específico con proyección, en este caso la industria del software y sus servicios asociados.

\subsection{Aprendizaje basado en Proyectos}

El Aprendizaje Basado en Proyecto - ABP - se destaca como complemento estratégico de la implementación del aprendizaje basado en competencias (UE, 2012), ya que permite que los estudiantes desarrollen competencias independientemente de la personalidad mediante la incorporación de escenarios reales en su estrategia de aprendizaje.

Con base en lo anterior la estrategia metodológica propuesta comprende el desarrollo de competencia mediante la implementación de proyectos pedagógicos integradores, en donde sus productos y entregables van de la mano con el desempeño de los aprendices en ambientes reales.

\subsection{Proyecto pedagógico integrador (PPI)}

El PPI, es una herramienta pedagógica y didáctica que permite a través del desarrollo de un proyecto, evidenciar en el alumno el desarrollo de competencias y capacidades; involucrando la 
planeación del proyecto y se concreta mediante productos de gestión; donde estudiantes y profesores pueden re-significar y re-contextualizar alrededor de éste.

El PPI aborda problemas del mundo real trabajando situaciones cercanas a las que tendrá el estudiante en su ejercicio profesional, enfocándose en un aprendizaje experimental (Efstratia, 2014). Lo anterior evidencia su fortaleza principal, ya que permite la reducción de tiempos de inducción en los estudiantes.

Los estudiantes desarrollan cada semestre un PPI centrado y dirigido por el módulo SOL (Ver Figura 1), el cual se enfoca en el desarrollo de una competencia principal por semestre.

El módulo sol, actúa como eje sobre el cual se define el proyecto y se integran los demás módulos que se trabajan durante cada semestre.

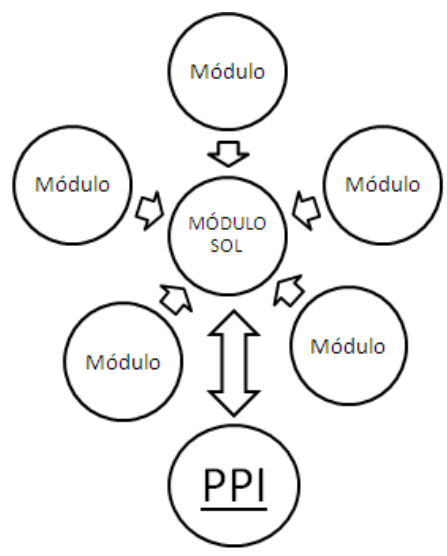

Fig. 1. PPI centrado en módulo Sol.

En la siguiente sección se presentan los trabajos relacionados con el aprendizaje a través de la formación en competencias, tomando como eje central la formación para el trabajo y entornos específicos de la industria.

\section{TRABAJOS RELACIONADOS}

El aprendizaje basado en proyectos facilita la adquisición de conocimiento y la validación de las competencias adquiridas, así mismo los docentes pasan a ser un integrante más del equipo de trabajo, sin embargo, como se menciona en Voronchenko et al.,(2015), el reto que se presenta es lograr realizar proyectos integradores con grupos multiculturales (ciudadanos del mundo) a partir de la práctica constante de la pedagogía de la tolerancia.

En (Gonçalves, 2014) se presenta un enfoque basado en proyectos complementado con aprendizaje activo (active-learning) con el fin de mejorar el aprendizaje del estudiante y preparar los futuros profesionales para su desempeño en sus prácticas laborales.

$\mathrm{Su}$ trabajo se centra en relacionar fuertemente la teoría con la práctica por medio del desarrollo de soluciones a problemas reales, similares a los que se enfrentará el estudiante en su contexto laboral.

En (Tasci, 2015) se preparan los estudiantes para su transición de la educación básica a la educación superior, tomando como elemento principal, la internacionalización de un conocimiento en común y su aplicación en ambientes escolares o universitarios, de esta menta se reduce el impacto social del estudiante al momento de cambiar su nivel educativo.

La aprendizaje de competencias no es ajeno a su contraparte en ambientes virtuales, ya que garantiza una cobertura en lugares donde no es posible la presencialidad, sin embargo, son varios los retos que se presentan (Bennani et al., 2012), como son: diseño y planeación de cursos, implementación de estrategias y contenidos, evaluación de procesos y validación de las competencias. No obstante, las investigaciones continúan con el fin de masificar el aprendizaje.

En cuanto a la formación para la industria del software, en (Indiramma, 2014) se resalta la importancia del trabajo en grupo y el beneficio social que acarrea la interacción entre distintos individuos, de esta manera se fomenta el pensamiento crítico, creativos e innovador.

Caso similar se presenta en (Song, 2011), que se presenta una reflexión alrededor de la inclusión de la formación por competencias en los currículos de desarrollo de talento en software, tomando la participación voluntaria de los estudiantes en proyecto de desarrollo de software.

Los trabajos mencionados anteriormente, demuestran la importancia del uso de un enfoque basado en proyectos en la formación por competencias, sin embargo carecen de un enfoque sistémico que permita la identificación y evaluación de los distintos elementos participantes y facilite así un mejoramiento continuo. 


\section{METODOLOGÍA PROPUESTA}

La metodología propuesta se compone por: descripción de elementos del PPI y el proceso de des arrollo.

\subsection{Elementos del PPI}

Estudiantes: Es el principal elemento por el cual el proyecto pedagógico integrador es implementado, ya que centra sus acciones el desarrollo de sus competencias.

Docentes: Desempeñan un rol de acompañante y asesor en cada uno de los proyectos. Así mismo facilita el avance de los proyectos mediante los saber impartidos en su clase.

Equipos: Como competencia transversal, el PPI busca el desarrollo de capacidades de trabajo en equipo. De esta manera el estudiante adquiere responsabilidades y desempeña roles dentro del desarrollo del proyecto.

Coordinación y gestión: Para un desarrollo apropiado del PPI, es necesario tener una control y gestión de la información generada, así como el monitoreo de los actores.

Módulos: Son el mecanismo por el cual se impaten los conocimientos y se definen los alcances del PPI.

Sector empresarial: Facilita el ecosistema donde el estudiante puede identificar problemáticas $y$ proponer soluciones. Así mismo ofrece la posibilidad de vivir experiencias en ambientes relaes, que permiten reducir el tiempo de inducción.

Proyectos: Evidencian el alcance de la competencia gracias a la definición de alcacnes y aporte de cada uno de los módulos.

Sistema de evaluación: Permite que se diseñen entregables como productos específicos. De esta manera es posible generar un impacto y beneficio directo sobre el usuario del proyecto.

Socializaciones: Momento evaluativo donde la comunidad académica, el sector empresarial y la docentes, validan el desarrollo de las competencias a través de la presentación del proyecto por parte de los equipos de trabajo.
Cabe señalar que los elementos previamente explicados permiten a los módulos ajustar pertinentemente sus contenidos en pro de la formación por competencias. Así pues, facilita la labor de actualización y mejoramiento de contenidos curriculares.

En la figura 2 se expone la interacción entre los distintos elementos, desde un punto de vista conceptual.

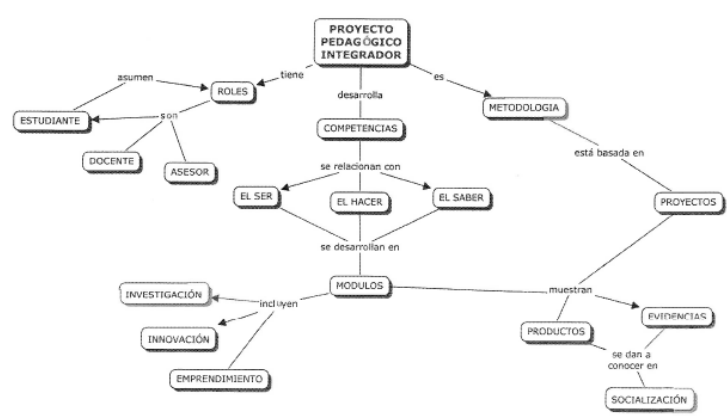

Fig. 2. Diagrama de interacción de los elementos del PPI.

El concepto central es el Proyecto Pedagógico Integrador, a partir de él se despliegan conceptos como: Metodología, Competencias y Roles. Lo que se busca es que la formación siempre este enfocada en el desarrollo de proyecto, teniendo en cuenta sus competencias y facilitando su participación en equipos de trabajos mediante los roles que desempeña.

\subsection{Proceso de desarrollo propuesto}

En la Figura 3 se presenta el proceso de desarrollo del PPI, el cual está compuesto por 4 fases a seguir:

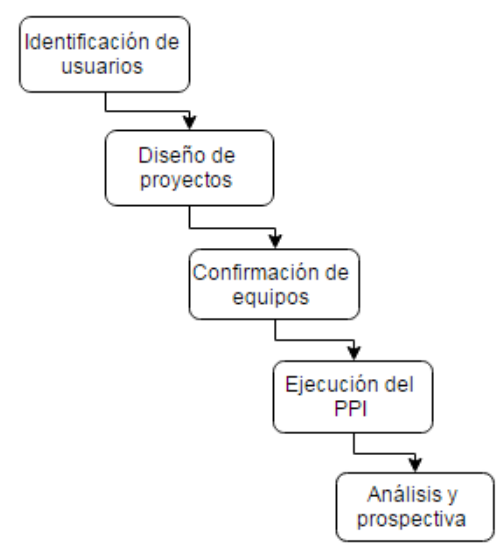

Fig. 3. Proceso de desarrollo del PPI. 
Fase 1: Identificación de usuarios: Al inicio del periodo académico, la coordinación del PPI, estudia las posibles empresas a impactar, teniendo en cuenta: pertinencia de las soluciones, facilidad y disposición.

Fase 2: Diseño de proyectos: Después de seleccionar las empresas y su disposición de trabajo, se realiza un estudio de las problemáticas relacionadas. A partir de ello se definen posibles soluciones y resultados de aprendizajes, insumo para el diseño de los proyectos.

Parte esencial del diseño de los proyectos es la definición de un cronograma de entregables, momentos evaluativos y socializaciones.

Los proyectos deben incluir criterios de evaluación de cada uno de sus resultados de aprendizaje. Para ello se emplean las matrices de evaluación o Rúbricas de evaluación (Brookhart, 2013).

Las rúbricas se caracterizan por la definición de criterios de evaluación de la competencia y sus niveles de alcance. Para el caso que nos ataña, se propone las siguientes escalas valorativas: Insuficiente, Aceptable, Bueno y Excelente. Cada una de ellas tiene asociada un valor numérico con el fin de obtener una calificación académica entre 0.0 y 5.0

Fase 3 - Conformación de equipos: Una vez definidos los proyectos, son redactados y presentados a los estudiantes. La conformación de los equipos debe cumplir con requisitos especiales, como son: (a) número máximo de integrantes es de 3 y un mínimo de 3, (b) los estudiantes deben compartir como mínimo el $50 \%$ de los módulos de su semestre.

Debido a la dinámica de los procesos académicos y curriculares, pueden presentarse casos excepcionales donde solo haya un estudiante; en estos casos se definen proyectos de aula con el acompañamiento de los profesores titulares.

Fase 4 - Ejecución del PPI: Los equipos de trabajo proceden al logro de los objetivos del proyecto. Como estrategia de desarrollo, los profesores de los módulos brindan asesorías extraclase, con el fin de obtener constante retroalimentación del desempeño de los equipos, así como en el direccionamiento técnico del mismo.

Es importante señalar que cada uno de los módulos aporta un producto en el desarrollo del PPI, de esta manera se hace necesario conservar la coherencia en las rúbricas de evaluación.

Parte de la ejecución del PPI, considera la actividad de evaluación y socialización, la cual busca divulgar los resultados obtenidos y dar a cada uno de los equipos una valoración cualitativa de su desempeño.

Fase 5 - Análisis y retroespectiva: Se analizan los resultados obtenidos basados en productos en términos de alcances, opinión de los usuarios y estudiantes. En esta fase se analizan los casos especiales y las soluciones aplicadas, para determinar su viabilidad futura.

\section{VALIDACIÓN Y ANÁLISIS DE RESULTADOS}

En esta sección se presenta la validación de la aproximación. El caso de estudio empleado se relaciona con el programa Técnico Profesional en Soporte de Sistemas de Información en su primer semestre. A partir de ello se presenta la descripción del PPI, las rúbricas definidas y los datos obtenidos de impacto.

\subsection{Descripción del PPI}

Descripción general: El equipo de trabajo debe identificar una institución educativa, con mínimo 5 equipos de cómputo (portátiles o de escritorio).

Los equipos de cómputo pueden tener instalado cualquier sistema operativo y estar conectados entre sí por medio de una red de datos. El proyecto pedagógico busca que el estudiante, sea capaz de:

- Realizar visitas a la institución por medio de un protocolo establecido.

- Levantar información de por lo menos 2 procesos. - Describir Infraestructura de hardware, software y redes que dan soporte a los procesos descritos. - Realizar un diagrama de flujo de datos de los procesos descritos.

-Proponer un prototipo de navegación de uno (1) de los procesos.

Módulos asociados. El PPI del primer semestre tiene como eje principal al módulo sol "Desarrollo de habilidades comunicativas y atención al cliente", siendo esta la competencia principal a desarrollar. En la Figura 4 se presentan los módulos relacionados. 


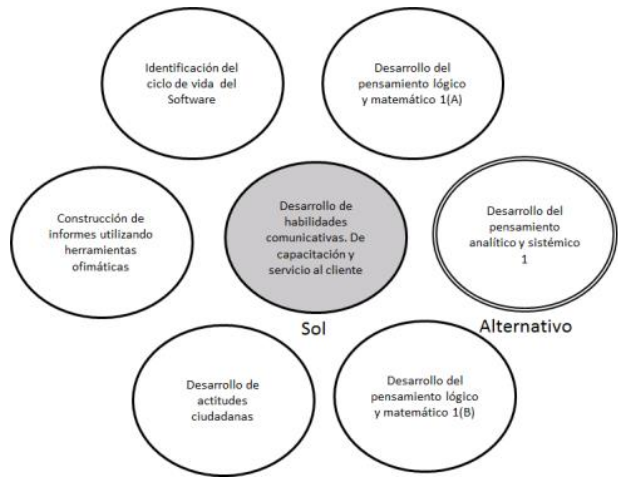

Fig. 4. Módulos asociados al PPI de primer semestre.

Productos asociados. El PPI del primer semestre tiene los siguientes productos asociados:

P1 - Protocolo de visitas y entrevista.

P2 - Análisis de visitas y entrevista.

P3 - Descripción de los procesos: Debe incluir software, hardware e infraestructura de red que les dan soporte.

P4 - Diagramas de flujo de cada uno de los procesos.

P5 - Prototipo de navegación: Programa hecho en consola relacionado con información de los procesos analizados.

P6 - Informe técnico PPI.

En la Tabla 1, se presenta el aporte de cada uno de los módulos del primer semestre al proyecto.

Tabla 1. Aporte por productos de cada uno de los módulos

\begin{tabular}{lc}
\hline \multicolumn{1}{c}{ Módulo } & Productos \\
\hline $\begin{array}{l}\text { Identificación del ciclo del } \\
\text { vida del software }\end{array}$ & $\mathrm{P} 4, \mathrm{P} 5, \mathrm{P} 6$ \\
\hline $\begin{array}{l}\text { Dllo de habilidades común. } \\
\text { y servicio al cliente }\end{array}$ & $\mathrm{P} 1, \mathrm{P} 2, \mathrm{P} 6$ \\
\hline $\begin{array}{l}\text { Dllo del pensamiento } \\
\text { analítico y sistémico 1 }\end{array}$ & $\mathrm{P} 4, \mathrm{P} 5, \mathrm{P} 6$ \\
\hline $\begin{array}{l}\text { Dllo del pensamiento lógico } \\
\text { y matemático 1A }\end{array}$ & $\mathrm{P} 2, \mathrm{P} 6$ \\
\hline $\begin{array}{l}\text { Dllo del pensamiento lógico } \\
\text { y matemático 1B }\end{array}$ & $\mathrm{P} 2, \mathrm{P} 6$ \\
\hline $\begin{array}{l}\text { Dllo de actitudes } \\
\text { ciudadanas }\end{array}$ & $\mathrm{P} 1, \mathrm{P} 6$ \\
\hline $\begin{array}{l}\text { Const. de informes } \\
\text { utilizando herr. informáticas }\end{array}$ & $\mathrm{P} 6$ \\
\hline
\end{tabular}

\subsection{Criterios de evaluación}

Los criterios de evaluación tienen en cuenta aspectos delsaber, hacer y del ser.
- Criterio 1: Conocimiento de la empresa.

- Criterio 2: Protocolo de visitas

- Criterio 3: Gestión del proyecto

- Criterio 4: Análisis de encuestas

- Criterio 5: Atención de clientes.

- Criterio 6: Expresión escrita

- Criterio 7: Apoyo audiovisual

- Criterio 8: Destrezas verbales.

Para el caso del criterio 3 (gestión del proyecto), se tienen las siguientes escalas de valoración:

Insuficiente: No se definieron roles ni responsabilidades. No existe un plan de trabajo y cronograma. Se evidencia problemas en el trabajo en grupo. Este criterio tiene un valor cuantitativo de cero $(0)$.

Aceptable: Se definieron roles y responsabilidades. No existe un plan de trabajo y cronograma. No se evidencia problemas en el trabajo en grupo. Este criterio tiene un valor cuantitativo de tres (3).

Bueno: Se definieron roles y responsabilidades. Existe un plan de trabajo y cronograma, pero no se está cumpliendo. Este criterio tiene un valor cuantitativo de cuatro (4).

Excelente: Se definieron roles y responsabilidades. Existe un plan de trabajo y cronograma. Está al día con el cronograma. Este criterio tiene un valor cuantitativo de cinco (5).

\subsection{Análisis de resultados}

En la siguiente tabla se presenta el número de proyectos realizados, así como las empresas impactadas.

\section{Tabla 2. Proyectos versus empresas impactadas}

\begin{tabular}{lcc}
\hline \multicolumn{1}{c}{ Semestre } & Proyectos & Empresas \\
\hline Primer semestre & 7 & 7 \\
\hline Segundo semestre & 6 & 4 \\
\hline Tercer semestre & 5 & 3 \\
\hline Cuarto semestre & 3 & 3 \\
\hline Quinto semestre & 3 & 1 \\
\hline Séptimo semestre & 2 & 1 \\
\hline
\end{tabular}

El número de proyectos no siempre es igual al número de empresas ya que, debido a la dinámica académica, algunos proyectos son diseñados para ser ejecutados como proyectos internos de aula. 
Es importante señalar que el perfil de desempeño del estudiante mantiene en constante validación de su pertinencia debido a su inmersión en ambientes reales de la industria.

\section{CONCLUS IONES}

Se presenta una aproximación metodológica que permita el diseño de proyectos pedagógicos integradores apropiados para el desempeño y formación basada en competencias de la industria del software.

La formación por competencias para el sector de la industria del software presenta una facilidad en su implementación mediante la aproximación basada en proyectos, ya que la industria ofrece la posibilidad de desarrollo de proyecto en ambientes reales de ejecución.

La participación de usuarios reales mejora la adquisición de saberes a partir de su ejemplificación en ambientes de validación correctos.

Como trabajo futuro se debe determinar una métrica para medir el desempeño de los estudiantes con base en el ambiente de ejecución, así mis mo se pretende el diseño de propuesta tecnológica enfocada hacia una aplicación distribuida que permita la gestión de la información, disponible para todos los actores en la formación basada en competencias.

\section{REFERENCIAS}

ACM. (2005). Computing Curricula 2005. Computer Science Final Report (September 30, 2005).

Bennani S., Idrissi M, Fadouli N., Yassine B., y Ouguengay, O. (2012). Online Project based learning driven by competencies: A systematic strategy proposal for assessment. Interactive Mobile and Computer Aided Learning (IMCL), 2012 Int. Conf. on , pp.92-97.

Brookhart S. (2013). How to Create and Use Rubrics for Formative Assessment and Grading.

Efstratia D. (2014) Experiential Education through Project Based Learning, Procedia - Social and Behavioral Sciences, Volume 152, 7 October 2014, pp. 1256-1260.

Gonçalves, S. (2014). Preparing Graduates for Professional Practice: Findings from a Case
Study of Project-based Learning (PBL) Procedia - Social and Behavioral Sciences, Volume 139, 22 August 2014, pp. 219-226.

Indiramma M. (2014). Project based learningTheoretical Foundation of Computation course. 2014 International Conference on Interactive Collaborative Learning (ICL). Dubai, UAE

Severin, E. (2011). Competencias para el siglo XXI: Cómo medirlas y cómo enseñarlas. Banco Interamericano de Desarrollo - Educación.

Spitzberg B. (1983). Communication competence as knowledge, skill and impression. Communication Education, 32(3): 323-329.

Tasc1 B. (2015). Project Based Learning from Elementary School to College, Tool: Architecture. Procedia - Social and Behavioral Sciences, Volume 186, 13 May 2015, pp.770775.

Turcio D. y Palacios, J. (2015). Experiencias en la enseñanza experimental basada en competencias. Educación Química, Volume 261. pp. $38-42$.

Unión Europea. Competencias para la inserción laboral. Guía de profesorado. Junio 2012.

Voronchenko T., Klimenko T. y Kostina I. (2015). Learning to Live in a Global World: ProjectBased Learning in Multicultural Student Groups as a Pedagogy of Tolerance Strategy. Procedia - Social and Behavioral Sciences, Volume 191, 2 June 2015, pp. 1489-1495. 